\title{
Três anos pós-LASIK em crianças anisométropes de 8 a 15 anos de idade
}

\author{
Three years after LASIK in anisometropic children from 8 to 15 yearsold
}

\author{
Belquiz R. Amaral Nassaralla ${ }^{1}$ \\ João J. Nassaralla Jr²
}

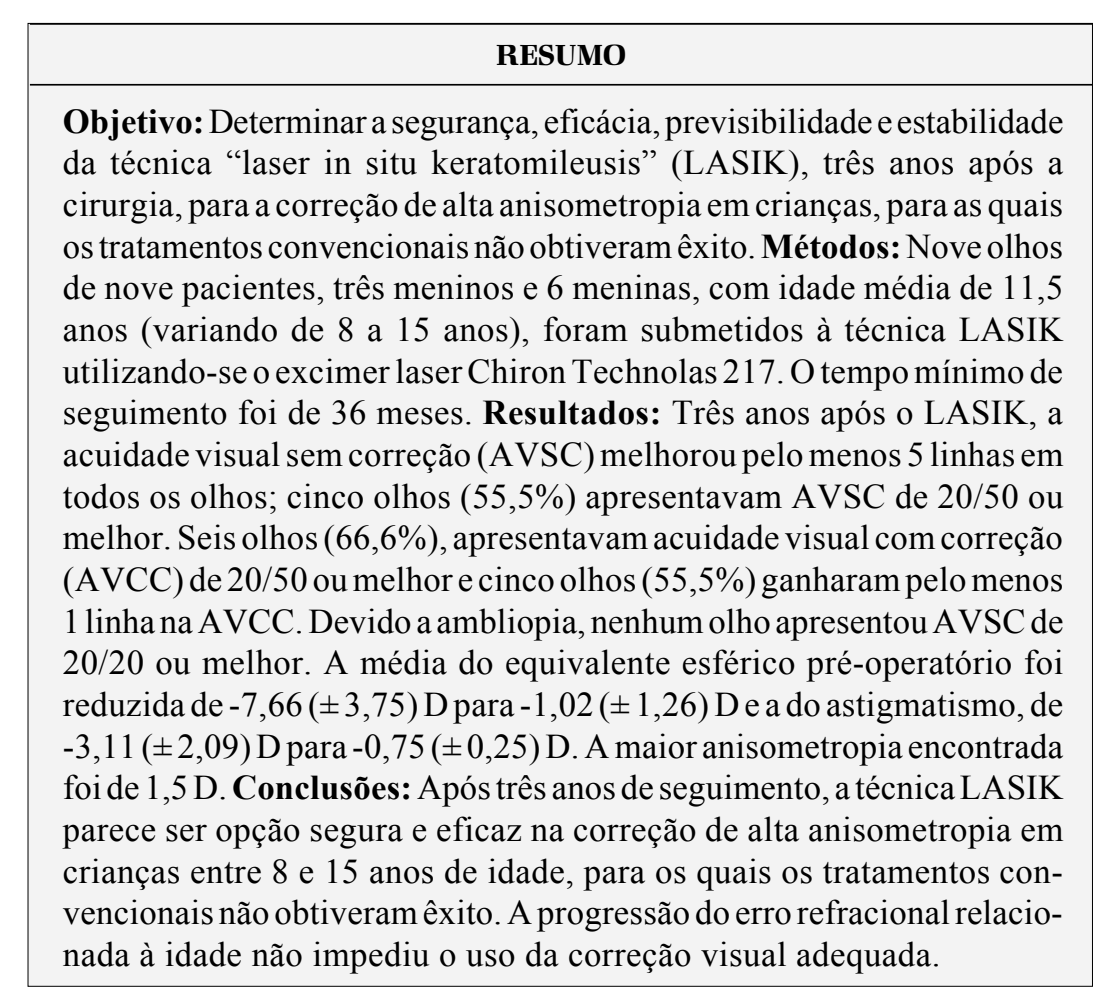

Descritores: Ceratomileuse assistida por excimer laser in situ; Miopia/cirurgia; Astigmatismo/ cirurgia; Resultado do tratamento; Adolescência; Criança

Trabalho realizado no Departamento de Córnea e Cirurgia Refrativa do Instituto de Olhos de Goiânia.

${ }^{1}$ Médica Oftalmologista do Departamento de Córnea e Cirurgia Refrativa do Instituto de Olhos de Goiânia (IOG); Doutora em Oftalmologia pela Universidade Federal de Minas Gerais.

${ }^{2}$ Médico Oftalmologista do Departamento de Retina e Vítreo do Instituto de Olhos de Goiânia (IOG); Doutorando em Oftalmologia pela Universidade Federal de Minas Gerais; Doutor em Ciências da Saúde pela Universidade de Brasília.

Endereço para correspondência: Rua $L \mathrm{n}^{\circ} 53,12^{\circ}$ andar, Setor Oeste - Goiânia (GO) CEP 74120-050 E-mail: nassaral@zaz.com.br

Recebido para publicação em 13.08.2001 Aceito para publicação em 12.06.2002

Nota Editorial: Pela análise deste trabalho e por sua anuência na divulgação desta nota, agradecemos à Dra. Claudia Maria Francesconi.

\section{INTRODUÇÃO}

Nos últimos anos, procedimentos cirúrgicos tais como a fotoceratectomia refrativa (PRK) e "laser in situ keratomileusis" (LASIK) têm sido usados com sucesso para tratar erros refracionais em adultos, tornando-os independentes de óculos e/ou lentes de contato ${ }^{(1-8)}$. Normalmente, a cirurgia refrativa é realizada em pacientes com mais de 18 anos de idade, após a estabilização da refração ${ }^{(1-3)}$. Com o aprimoramento das técnicas, é natural que queiramos ampliar seu campo de ação e beneficiar um número maior de pessoas, inclusive crianças e adolescentes com altas anisometropias, para as quais o tratamento convencional, com óculos ou lentes de contato, não obteve sucesso.

Alguns estudos mostram bons resultados da técnica PRK em crianças $^{(9-11)}$; outros mais recentes obtiveram sucesso com a LASIK ${ }^{(12-14)}$ em pacientes com menos de 18 anos de idade, principalmente em casos de alta anisometropia. Infelizmente, o curto período de seguimento destes pacientes (entre 3 e 12 meses) nos privaram de informações importantes que podem ocorrer com a progressão da idade.
\end{abstract}


Anisometropia é a diferença de erro refracional entre os dois olhos de um mesmo indivíduo. Considera-se que ela existe quando a diferença é superior a $1,00 \mathrm{D}$ no componente esférico e/ou $0,75 \mathrm{D}$, no valor cilíndrico ${ }^{(15)}$. Quando maior que 3,0 D, esta assimetria pode causar sintomas de astenopia tais como: queimação, ardência, prurido, lacrimejamento, diplopia, fotofobia, cefaléia e visão borrada. Estes sintomas são produzidos através de dois mecanismos: diferença no tamanho das imagens retinianas (aniseiconia) e deslocamento prismático das imagens causado pelas lentes dos óculos. A anisometropia é uma das principais causas de ambliopia monocular e o grau de ambliopia está fortemente relacionado à diferença refracional entre os olhos ${ }^{(16)}$. Alguns fatores tais como a baixa idade, capacidade de fusão e história prévia do uso de óculos, podem facilitar a tolerância do paciente à anisometropia. Para outros, a correção total com óculos pode ser intolerável e a hipocorreção ou o uso de lentes de contato, são as melhores opções ${ }^{(17)}$. Infelizmente, a imagem borrada do olho hipocorrigido dificulta a visão binocular e, para pacientes muito jovens, as lentes de contato são de difícil manuseio e adaptação ${ }^{(15)}$. Nestes casos de intolerância às terapias convencionais, restam poucas alternativas; portanto, a cirurgia refrativa é uma opção que não deveria ser descartada.

A indicação deste tipo de cirurgia para indivíduos tão jovens não é uma opção fácil e por isso mesmo, bastante controversa. A coleta de dados precisos antes da cirurgia é mais difícil, os riscos per-operatórios são aumentados pela menor cooperação e, além disso, existem as alterações refracionais que continuarão a ocorrer até o início da idade adulta. Diferentemente dos adultos, para estes pacientes, o mais importante não é torná-los independente de óculos, mas sim reduzir a anisometropia, permitir o uso da correção visual adequada e favorecer a visão binocular.

Em estudo anterior ${ }^{(18)}$, acompanhamos e avaliamos este grupo de pacientes por um período de 1 ano. Nossos dados foram encorajadores e mostraram ser o LASIK uma técnica segura e eficaz para este seleto grupo de pacientes. Porém, a limitada informação disponível na literatura, o curto período de seguimento, a pouca experiência com esta técnica em pacientes com idade inferior a 18 anos e a provável progressão do erro refracional até a maturidade, nos levaram a continuar acompanhando estes jovens pacientes e analisando os dados encontrados ao longo do tempo. Neste estudo, três anos após a cirurgia, reavaliamos a segurança, eficácia, previsibilidade e estabilidade da técnica "laser in situ keratomileusis" para a correção de alta anisometropia em crianças e adolescentes entre 8 e 15 anos de idade.

\section{MÉTODOS}

Em um estudo prospectivo, nove olhos de 9 pacientes, 3 do sexo masculino e 6 do sexo feminino, com alta anisometropia $(>3,5 \mathrm{D})$ foram submetidos à técnica LASIK para correção de alto erro refracional unilateral. A idade média dos pacientes era de 11,5 anos, variando entre 8 e 15 anos de idade. Os pacientes foram selecionados após demanda espontânea ao ambulatório do Instituto de Olhos de Goiânia. A participação no estudo foi proposta a todos os pacientes e seus responsáveis após a devida orientação quanto aos riscos relacionados à cirurgia, a provável alteração refracional relacionada à progressão da idade e sobre a limitada experiência com a técnica LASIK em pacientes com idade inferior a 18 anos. O responsável por cada paciente assinou um termo de consentimento livre e esclarecido, segundo protocolo aprovado pela Comissão de Ética do Instituto de Olhos de Goiânia.

O critério de inclusão foi: alta anisometropia $(>3,5 \mathrm{D})$ secundária a alta miopia ou alto astigmatismo miópico, intolerância ao uso de óculos e/ou lentes de contato, ausência de doença sistêmica, paciente calmo e cooperativo, comparecimento a todos os exames solicitados por um período mínimo de três anos, família esclarecida sobre o procedimento e colaborativa no sentido de executar as instruções pós-operatórias.

As cirurgias foram realizadas com o excimer laser Technolas 217-C e o microceratótomo Automated Corneal Shaper (Bausch \& Lomb/Chiron Vision, Claremont, CA, EUA) entre janeiro e agosto de 1998. A técnica cirúrgica foi basicamente a mesma utilizada para pacientes adultos ${ }^{(3,6)}$, exceto quanto ao uso do oclusor de plástico no pós-operatório. Normalmente, o oclusor é mantido sobre o olho operado nas primeiras $24 \mathrm{~h}$ e depois, só para dormir, durante 8 dias. Neste grupo de pacientes, o oclusor foi mantido ininterruptamente sobre o olho, durante os primeiros 8 dias de pós-operatório. Não houve necessidade de anestesia geral em nenhum dos pacientes.

Os diagnósticos pré-operatórios foram: ambliopia anisometrópica secundária à alta miopia e/ou alto astigmatismo miópico, unilateral, congênito em seis olhos $(66,6 \%)$; alto erro refracional residual secundário a facectomia pós-trauma em dois olhos $(22,2 \%)$; e transplante de córnea pós-trauma em um olho (11,1\%), (Tabela 1). O tempo decorrido entre a cirurgia de LASIK após o transplante de córnea foi de 24 meses e após a cirurgia de catarata, de 16 meses em 1 olho e 13 meses em outro.

Os exames pré-operatórios incluíram: histórico médico ocular, avaliação ortóptica, medida da AVSC e AVCC, topografia corneana, ceratometria, paquimetria ultra-sônica, refração manifesta e cicloplegiada, tonometria, biomicroscopia, microscopia especular da córnea e oftalmoscopia binocular indireta com depressão escleral circunferencial. A midríase foi realizada com colírio de ciclopentolato a $1 \%$ e fenilefrina a $10 \%$. Os exames do segmento posterior foram realizados por um mesmo médico oftalmologista, especialista em retina $(J J N)$ que procurou identificar possíveis lesões na periferia da retina, principalmente nos pacientes portadores de alta miopia.

Antes da cirurgia, o erro refracional a ser corrigido era digitado no computador do laser. Em todos os casos, operou-se a refração cicloplegiada do olho com o maior erro refracional. A faixa de miopia a ser operada era semelhante àquela tratada satisfatoriamente em adultos, isto é, miopia até -12,00D, depen- 


\begin{tabular}{|c|c|c|c|c|c|c|}
\hline \multicolumn{7}{|c|}{ Tabela 1. Dados Pré-LASIK } \\
\hline Caso & Sexo & Idade & Olho & Diagnóstico & $\begin{array}{l}\text { Refração estática } \\
\text { do olho operado }\end{array}$ & $\begin{array}{l}\text { Refração estática } \\
\text { do olho adelfo }\end{array}$ \\
\hline 1 & Fem. & 8 & $\mathrm{D}$ & Ambl. Anisomet. & $-10,00-2,00 \times 100$ & $-0,50-1,50 \times 90$ \\
\hline 2 & Fem. & 9 & $\mathrm{D}$ & Ambl. Anisomet. & $-8,00-5,00 \times 15$ & Plano $-2,50 \times 180$ \\
\hline 3 & Fem. & 9 & $\mathrm{D}$ & Ambl. Anisomet. & $-6,00-0,50 \times 110$ & Plano \\
\hline 4 & Masc. & 10 & $E$ & Pós EECC + LIO & $-0,50-5,50 \times 160$ & $+1,00$ \\
\hline 5 & Masc. & 12 & $\mathrm{D}$ & Pós EECC + LIO & Plano $-5,00 \times 175$ & $+1,25$ \\
\hline 6 & Fem. & 12 & $E$ & Ambl. Anisomet. & $-6,75-2,00 \times 180$ & $-1,25-0,50 \times 15$ \\
\hline 7 & Masc. & 14 & $E$ & Pós TC & $-1,50-6,00 \times 150$ & $+0,50$ \\
\hline 8 & Fem. & 15 & $\mathrm{D}$ & Ambl. Anisomet. & $-10,25$ & $-0,25$ \\
\hline 9 & Fem. & 15 & $E$ & Ambl. Anisomet. & $-13,00$ & $-1,00-0,50 \times 135$ \\
\hline
\end{tabular}

dendo da espessura corneana e do tamanho da pupila do paciente $^{(5)}$.

Apenas o paciente 9, apresentava erro refracional ligeiramente superior a esta faixa (Tabela 1).

Todos os pacientes foram pré-medicados com sedativo oral (diazepam $5 \mathrm{mg}$ ) 30 minutos antes da cirurgia, sendo bastante cooperativos. Dois pacientes, um do sexo feminino e outro do sexo masculino, respectivamente com 8 anos e 9 anos de idade, foram excluídos do estudo e desaconselhados a realizar a cirurgia, ainda durante os exames pré-operatórios, por demonstrarem nervosismo e pouca colaboração. Apenas os pacientes 4 e 5 , ambos do sexo masculino, solicitaram acompanhamento dos pais durante o procedimento.

Com o objetivo de manter o paciente tranqüilo, mantivemos a comunicação oral com o paciente durante toda a cirurgia. Os pacientes foram examinados após 1 dia e 7 dias da cirurgia e depois com 1, 3, 6, 9, 12, 24 e 36 meses. A partir de um mês da cirurgia, os exames incluíram: medida da AVSC e AVCC, topografia corneana, ceratometria, paquimetria ultrasônica, microscopia especular da córnea, refração manifesta e cicloplegiada, tonometria, biomicroscopia, microscopia especular da córnea e fundoscopia. Em cada visita, os pacientes eram questionados sobre sintomas tais como "glare", flutuação da visão, ardor, sensação de corpo estranho e fotofobia. No exame de 36 meses, a satisfação do paciente foi avaliada através de questionário. Inicialmente foi perguntado ao paciente e seus responsáveis, se eles concordariam com a cirurgia novamente e se a indicariam a outras crianças na mesma situação. O questionário também incluía perguntas sobre a satisfação com a AVSC e AVCC após o procedimento e sobre a evolução dos sintomas relacionados à anisometropia, referidos antes da cirurgia, tais como queimação, ardência, prurido, lacrimejamento, diplopia, fotofobia, cefaléia e visão borrada.

O método estatístico utilizado para a comparação das medidas pré-operatórias e pós-operatórias até os 36 meses de seguimento foi à análise de variância para medidas repetidas, já que as variáveis estudadas tinham distribuição normal. Foram considerados estatisticamente significativos os achados com $\mathrm{p}<0,05$.

\section{RESULTADOS}

Antes da cirurgia, 4 pacientes não aceitavam qualquer tipo de correção visual e 5 estavam hipocorrigidos unilateralmente com óculos.

O olho adelfo, não operado, apresentava acuidade visual sem correção (AVSC) maior ou igual a 20/40 e com correção (AVCC) igual a 20/20, em todos os pacientes. A média do equivalente esférico no olho a ser operado, sob cicloplegia, era de -7,66 ( $\pm 3,75) \mathrm{D}$ (variando de -2,50 a -13,00D), (Tabela 2). A AVSC era menor ou igual a $20 / 300$ e a AVCC, maior ou igual a 20/150 (variando de 20/150 a 20/30), (Tabela 3). A média da anisometropia encontrada foi de 7,25 $( \pm 2,81) \mathrm{D}$, variando entre 3,75 e 11,75 D, (Tabela 4).

Nenhum paciente apresentou complicações per-operatórias ou dificuldade em cooperar durante o procedimento. Após a cirurgia, as complicações encontradas foram: crescimento epitelial na interface em 1 paciente $(11,1 \%)$ e pregas na membrana de Bowman em 1 paciente $(11,1 \%)$. O crescimento epitelial ficou limitado a 1,0 mm da margem do "flap" e não necessitou de tratamento. As pregas na membrana de Bowman desapareceram completamente após um mês.

Um ano após a cirurgia, todas as córneas apresentavam-se transparentes, sem deslocamento do disco e a cicatriz da ceratotomia feita com o microceratótomo, era dificilmente visualizada, mesmo sob lâmpada de fenda.

\begin{tabular}{|lccccc|}
\hline \multicolumn{7}{|c|}{ Tabela 2. Equivalente Esférico $\left(^{*}\right)$ Pré- e Pós-LASIK } \\
Caso & Pré & $\mathbf{3}$ meses & $\mathbf{1 2}$ meses & 24 meses & $\mathbf{3 6}$ meses \\
1 & $-11,00$ & $-0,75$ & $-1,50$ & $-1,75$ & $-2,25$ \\
2 & $-10,50$ & $-1,00$ & $-1,00$ & $-1,75$ & $-2,00$ \\
3 & $-6,25$ & $+0,75$ & $+0,50$ & $-0,50$ & $-0,75$ \\
4 & $-3,25$ & $+0,75$ & $+0,75$ & $+0,50$ & $+0,75$ \\
5 & $-2,50$ & $-0,50$ & $-0,50$ & $-0,25$ & $-0,25$ \\
6 & $-7,75$ & $-0,25$ & $-1,00$ & $-1,50$ & $-2,00$ \\
7 & $-4,50$ & $+0,75$ & $+0,50$ & Plano & Plano \\
8 & $-10,25$ & $+2,50$ & $+2,00$ & $+1,00$ & Plano \\
9 & $-13,00$ & $-0,75$ & $-1,75$ & $-2,25$ & $-2,75$ \\
(*) Refração cicloplegiada $_{7}^{7}$ \\
\hline
\end{tabular}




\begin{tabular}{|c|c|c|c|c|c|c|c|c|c|c|}
\hline \multicolumn{11}{|c|}{ Tabela 3. Acuidade Visual Pré- e Pós-LASIK } \\
\hline \multirow{2}{*}{ Caso } & \multicolumn{2}{|c|}{ Pré } & \multicolumn{2}{|c|}{3 meses } & \multicolumn{2}{|c|}{12 meses } & \multicolumn{2}{|c|}{24 meses } & \multicolumn{2}{|c|}{36 meses } \\
\hline & SC & CC & SC & CC & SC & CC & SC & CC & SC & CC \\
\hline 1 & $20 / 800$ & $20 / 80$ & $20 / 80$ & $20 / 60$ & $20 / 80$ & $20 / 60$ & $20 / 80$ & $20 / 60$ & $20 / 100$ & $20 / 60$ \\
\hline 2 & $20 / 800$ & $20 / 150$ & $20 / 150$ & $20 / 150$ & $20 / 150$ & $20 / 150$ & $20 / 150$ & $20 / 150$ & $20 / 200$ & $20 / 150$ \\
\hline 3 & $20 / 400$ & $20 / 60$ & $20 / 40$ & $20 / 40$ & $20 / 40$ & $20 / 40$ & $20 / 40$ & $20 / 40$ & $20 / 50$ & $20 / 40$ \\
\hline 4 & $20 / 400$ & $20 / 40$ & $20 / 30$ & $20 / 30$ & $20 / 30$ & $20 / 30$ & $20 / 30$ & $20 / 30$ & $20 / 30$ & $20 / 30$ \\
\hline 5 & $20 / 400$ & $20 / 30$ & $20 / 30$ & $20 / 25$ & $20 / 30$ & $20 / 25$ & $20 / 25$ & $20 / 25$ & $20 / 25$ & $20 / 25$ \\
\hline 6 & $20 / 800$ & $20 / 40$ & $20 / 50$ & $20 / 40$ & $20 / 50$ & $20 / 40$ & $20 / 50$ & $20 / 40$ & $20 / 60$ & $20 / 40$ \\
\hline 7 & $20 / 300$ & $20 / 50$ & $20 / 50$ & $20 / 50$ & $20 / 50$ & $20 / 50$ & $20 / 50$ & $20 / 50$ & $20 / 50$ & $20 / 50$ \\
\hline 8 & $20 / 800$ & $20 / 60$ & $20 / 70$ & $20 / 50$ & $20 / 70$ & $20 / 50$ & $20 / 60$ & $20 / 50$ & $20 / 50$ & $20 / 50$ \\
\hline 9 & $20 / 1200$ & $20 / 150$ & $20 / 150$ & $20 / 150$ & $20 / 150$ & $20 / 150$ & $20 / 150$ & $20 / 150$ & $20 / 200$ & $20 / 150$ \\
\hline
\end{tabular}

\begin{tabular}{|lccccc|}
\hline \multicolumn{7}{c|}{ Tabela 4. Anisometropia Pré- e Pós- LASIK } \\
Caso & Pré & $\mathbf{3}$ meses & $\mathbf{1 2}$ meses & 24 meses & 36 meses \\
1 & 9,75 & 0,50 & 0,25 & 0,25 & 0,25 \\
2 & 8,25 & 0 & 0,50 & 0,25 & 0 \\
3 & 6,25 & 0,75 & 1,00 & 0,50 & 0,75 \\
4 & 4,25 & 0,25 & 0,25 & 0,50 & 0,25 \\
5 & 3,75 & 1,75 & 1,75 & 1,50 & 1,50 \\
6 & 6,25 & 1,50 & 1,00 & 0,75 & 0,50 \\
7 & 5,00 & 0,25 & 0 & 0,50 & 0,50 \\
8 & 10,00 & 2,75 & 2,50 & 1,75 & 1,00 \\
9 & 11,75 & 0,75 & 0 & 0,25 & 0 \\
\hline
\end{tabular}

Um ano após a cirurgia, a média do equivalente esférico pré-operatório foi reduzida de $-7,66( \pm 3,75) \mathrm{D}$ (variando de $-2,50$ a $-13,00 \mathrm{D}$ ) para $-0,22( \pm 0,89) \mathrm{D}$ (variando de $+2,00 \mathrm{a}-1,75$ $\mathrm{D}),(\mathrm{p}=0,0001)$, e a média do astigmatismo pré-operatório foi reduzida de $-3,11( \pm 2,09) \mathrm{D}$ (variando entre 0 e $-6,00 \mathrm{D}$ ) para $-0,69( \pm 0,25) \mathrm{D}$ (variando entre $0 \mathrm{e}-1,50 \mathrm{D})$. Três olhos $(33,3 \%)$ ficaram entre $\pm 0,50 \mathrm{D}$ da emetropia e seis olhos $(66,6 \%)$, ficaram entre $\pm 1,00 \mathrm{D}$. Três anos após a cirurgia, a média do equivalente esférico era de $-1,02( \pm 1,26) \mathrm{D}$ (variando entre $+0,75 \mathrm{D}$ e $-2,75 \mathrm{D}$ ), (Tabela 2 ) e a do astigmatismo era de $-0,75$ $( \pm 0,25) \mathrm{D}$ (variando de 0 a $-1,75 \mathrm{D})$.

Para avaliar a estabilidade do resultado cirúrgico, comparamos os dados encontrados após 3 e 36 meses após a cirurgia. Sete pacientes $(77,7 \%)$ incluindo os $n^{\circ}$ s 1, 2, 3, 6, 7, 8 e 9, apresentaram tendência à progressão da miopia e/ou regressão do efeito da cirurgia, com média de $-1,3 \mathrm{D}$ (variando de $0 \mathrm{a}$ $-2,75 \mathrm{D}$ ) no equivalente esfércio, (Tabela 2 ).

A média da anisometropia pré-operatória caiu de 7,25 $( \pm 2,81) \mathrm{D}$ (variando de 3,75 a $11,75 \mathrm{D}$ ) para $0,47( \pm 0,48) \mathrm{D}$ (variando de 0 a 1,5 D) 36 meses após a cirurgia, (Tabela 4).

Os exames de topografia corneana mostraram boa centralização da ablação (Figura 1). Em todos os olhos foi observada redução no poder refrativo central correspondente às alterações refrativas alcançadas. A figura 1 mostra uma série de mapas topográficos obtidos antes (a), após 3 meses (b), após 1 ano (c) e após três anos (d) da LASIK no paciente 6. Antes da cirurgia, o equivalente esférico do olho direito (operado) era $-7,75 \mathrm{D}$ e no olho esquerdo (não operado), -1,50 D. Após a cirurgia, o equivalente esférico do olho operado era $-0,25 \mathrm{D}$ aos 3 meses, -1,00 D aos 12 meses e -1,75 aos 3 anos.

Três meses após a cirurgia, pela tabela de Snellen, todos os pacientes apresentaram ganho de 5 linhas ou mais na AVSC. Três olhos (33,3\%) apresentavam AVSC de 20/40 ou melhor. Devido à alta incidência de ambliopia neste grupo de pacientes, nenhum olho apresentou AVCC de 20/20 ou melhor. Seis pacientes $(66,6 \%)$ apresentaram AVCC maior ou igual a $20 / 50$ e $4(44,4 \%)$, de 20/40 ou melhor. Cinco pacientes $(55,5 \%)$ apresentaram ganho de pelo menos 1 linha de visão na AVCC e $4(44,4 \%)$, mantiveram-na inalterada. Nos 3 pacientes com até 9 anos de idade ( ${ }^{\circ} 1,2$ e 3 ), foi feito tratamento oclusivo durante 1 ano para tentar melhorar a ambliopia. Dois deles apresentaram ganho de 2 linhas de visão na AVCC após 3 meses da cirurgia, resultado que se manteve inalterado durante os 3 anos de seguimento. Nenhum paciente perdeu linha de visão na $\mathrm{AV}$ com ou sem correção.

Após 12 meses, pela tabela de Snellen, a AVSC manteve o ganho de pelo menos 5 linhas de visão em todos os olhos. Três olhos $(33,3 \%)$ apresentavam AVSC de $20 / 40$ ou melhor. A AVCC não sofreu alteração em 4 olhos $(44,4 \%)$ e melhorou em 5 olhos $(55,5 \%)$ em pelo menos 1 linha na tabela de Snellen. Quatro olhos (44,4\%) apresentavam AVCC de 20/40 ou melhor.

Três anos após a cirurgia, 2 pacientes $(22,2 \%)$ apresentavam AVSC de 20/40 ou melhor e quatro (44,4\%) apresentavam AVCC de 20/40 ou melhor. Comparando os resultados de $3 \mathrm{com}$ 36 meses após a cirurgia, pela tabela de Snellen, nenhum dos olhos estudados apresentou alteração na AVCC (Tabela 3).

Antes da cirurgia, a média da contagem endotelial era de $2.211 \pm 160 \mathrm{cel} / \mathrm{mm}^{2}$ (variando de $1.400 \mathrm{a} 2.700 \mathrm{cel} / \mathrm{mm}^{2}$ ). Após 6 meses, $2.180 \pm 120$ células $/ \mathrm{mm}^{2}$ (variando de 1.390 a $2.690 \mathrm{cel} /$ $\mathrm{mm}^{2}$ ) e com 36 meses, $2.152 \pm 80 \mathrm{cel} / \mathrm{mm}^{2}$ (variando de $1.400 \mathrm{a}$ $2.300 \mathrm{cel} / \mathrm{mm}^{2}$ ). A percentagem de perda celular, 6 meses após a cirurgia, foi de $1,4 \%$ e após 36 meses, de $2,67 \%$.

A média da paquimetria central antes da cirurgia era de 555 $\pm 32 \mu \mathrm{m}$ (variando entre 525 e $598 \mu \mathrm{m}$ ). Seis meses após a cirurgia, era de $442 \pm 38 \mu \mathrm{m}$ (variando de 380 a $476 \mu \mathrm{m}$ ) e de 456 $\pm 30 \mu \mathrm{m}$ (variando de $390 \mathrm{a} 495 \mu \mathrm{m}$ ) após 36 meses. A ablação corneana variou de 75 a $160 \mu \mathrm{m}$. 


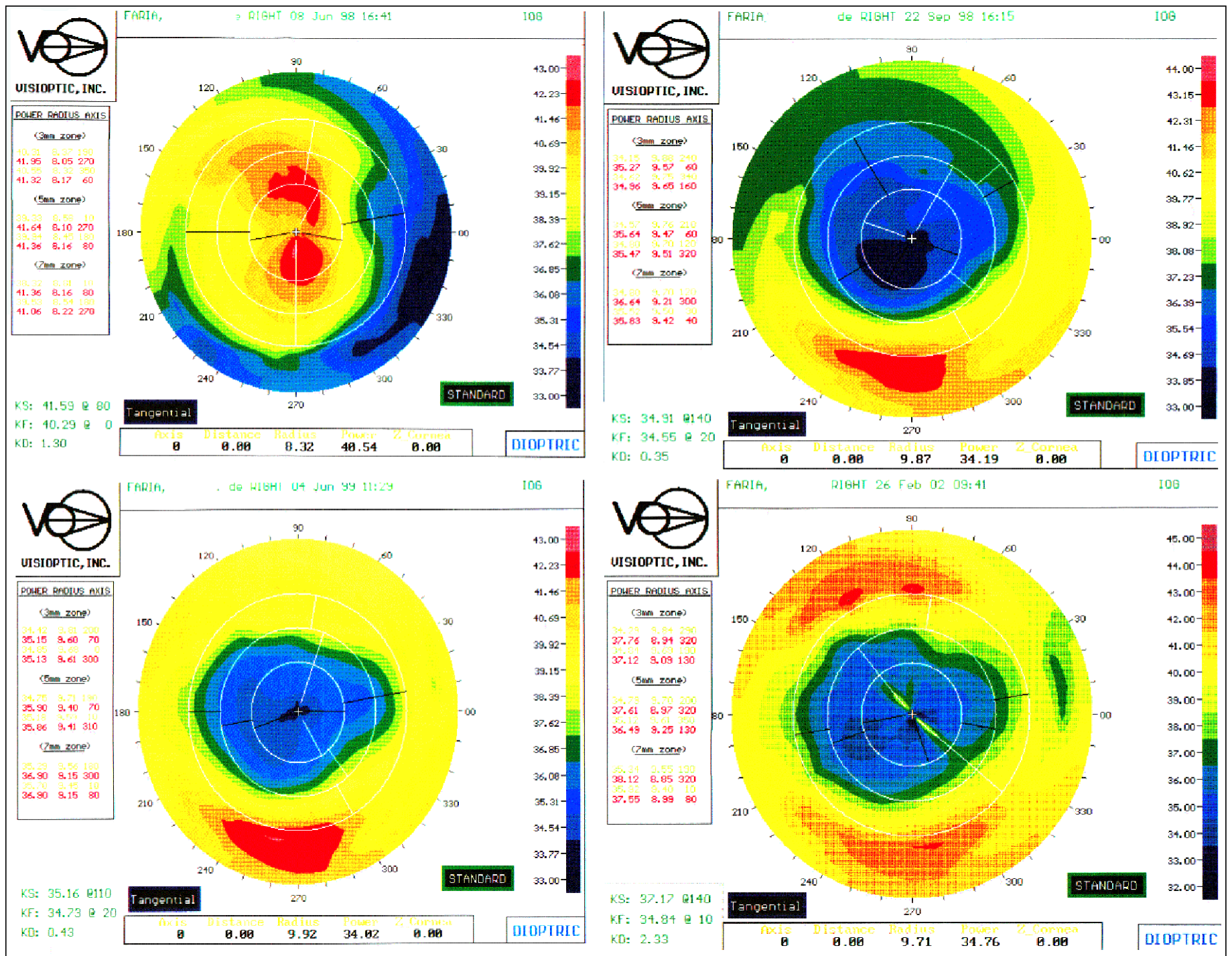

Figura 1 - Mapas topográficos obtidos antes (a), após 3 meses (b), após 1 ano (c) e após três anos (d) da cirurgia na paciente no 6

Não houve redução significativa $(\mathrm{p}>0,05)$ na média da pressão intra-ocular, que decresceu de 13,0 $\pm 1,5$ (variando de 11 a 14) $\mathrm{mmHg}$ antes da cirurgia para $12,1 \pm 1,0$ (variando de 11 a 13) $\mathrm{mmHg}$ após 6 meses e 11,0 $\pm 1,0$ (variando de 10 a 12) $\mathrm{mmHg}$ após 36 meses.

\section{DISCUSSÃO}

A eficácia da técnica LASIK, para este grupo de pacientes, foi avaliada pelo seguinte critério: redução significativa do erro refracional, redução da anisometropia para níveis que permitissem o uso da correção visual adequada, melhora da acuidade visual e melhora da qualidade de vida.

Optamos pela anestesia tópica para todos os pacientes por termos observado que apesar de jovens, mesmo os 3 pacientes com menos de 10 anos de idade, nos pareceram calmos e compreensivos o suficiente para colaborar durante o proce- dimento. Em caso de criança com menos idade ou pouco colaborativa, concordamos com outros autores ${ }^{(9-14)}$ sugerindo $o$ uso da anestesia geral. Infelizmente, com a anestesia geral, a centralização se torna mais difícil, mesmo que o cirurgião utilize recursos já descritos tais como: fixar o olho durante a ablação, usar colírio de pilocarpina no pré-operatório ou centralizar a área de ablação pelo limbo ${ }^{(11)}$. Além disso, existe o risco de ocorrer vazamento de gás durante a anestesia, o que pode interferir no funcionamento do laser, principalmente quando se usa óxido nitroso ${ }^{(14)}$.

A córnea atinge o tamanho adulto por volta de dois anos de idade. A miopia geralmente aparece entre 6 e 9 anos e progride durante a adolescência, com alterações mais acentuadas durante a puberdade ${ }^{(1-3)}$. Por isso, a cirurgia refrativa é normalmente adiada até a estabilização da refração, o que ocorre no início da idade adulta. No entanto, concordamos com outros autores ${ }^{(9-14)}$ cujos trabalhos mostram que crianças portadoras de alta anisometropia, que não respondem bem ao 
tratamento clínico convencional, podem ser beneficiadas pela cirurgia refrativa.

Apesar de alguns estudos ${ }^{(9-11)}$ sugerirem que a técnica PRK é relativamente segura e eficaz na redução de altos erros refracionais em crianças, ela ainda apresenta limitações tais como: desconforto pós-operatório, demora na recuperação e estabilização da acuidade visual, regressão do efeito obtido pela correção cirúrgica, formação de "haze" e perda de linhas na acuidade visual corrigida. Por outro lado, a recuperação visual mais rápida, menor desconforto pós-operatório, melhor estabilidade, ausência de "haze" e facilidade para reoperação, têm levado outros autores ${ }^{(12-14)}$ a preferir a técnica LASIK em vez da PRK, no tratamento da ametropia.

Os dados encontrados neste estudo mostram que em todos os pacientes houve redução da anisometropia a níveis que permitissem o uso da correção visual adequada.

Antes da cirurgia refrativa, um exame refracional preciso é muito importante e especialmente em crianças, esta não é uma tarefa fácil. Em casos de cirurgia ocular prévia, principalmente nos casos submetidos ao transplante penetrante de córnea, deve-se aguardar a estabilização do enxerto após a remoção dos pontos, para só depois proceder aos exames ${ }^{(6)}$. No paciente 7 , a técnica LASIK foi realizada 2 anos após o transplante penetrante e 6 meses após a remoção das suturas. Em estudo anterior sobre LASIK após transplante de córnea ${ }^{(6)}$, observamos que este espaço de tempo é suficiente para que a refração se estabilize, o que coincide com o relato de outros autores $^{(7-8)}$. Alguns autores ${ }^{(19)}$ sugeriram que, após o transplante de córnea, a técnica LASIK seja realizada em dois tempos cirúrgicos: no primeiro, o disco corneano é confeccionado pelo microceratótomo e reposicionado. Aguarda-se 1 ou 2 semanas até a estabilização da refração, principalmente do componente cilíndrico, e só então, procede-se à ablação do leito estromal com o laser. Apesar de não existirem dados estatísticos sobre as vantagens desta variação da técnica, é possível que ela melhore os resultados, porém, requer dois procedimentos, cada um com riscos de complicações, incluindo rejeição do enxerto e deiscência da ferida. Acreditamos que, se a ceratotomia e a ablação são realizadas em um mesmo tempo cirúrgico, o paciente tem a chance de ser submetido a apenas um procedimento cirúrgico, o que é muito importante, principalmente quando nos referimos a crianças e adolescentes. Além disso, fazendo a correção em um único tempo, caso ocorra erro refracional residual significativo, e havendo espessura corneana residual suficiente, um segundo procedimento pode ainda ser realizado, conforme apresentamos em estudo anterior ${ }^{(16)}$.

Quanto aos riscos relacionados à cirurgia, nossa preocupação era semelhante àquela descrita em outros estudos ${ }^{(7-8)}$ para pacientes adultos, que ressalta o risco de deiscência da cicatriz cirúrgica nos casos submetidos à cirurgia ocular prévia. Deiscências após transplante de córnea ou cirurgia de catarata são complicações que podem ocorrer mesmo após anos da cirurgia, especialmente quando a pressão intra-ocular é elevada a níveis em torno de $65 \mathrm{mmHg}^{(20)}$. Por isso, tentamos reduzir ao máximo o tempo de sucção. Nesta série de pacientes, a elevação da pressão intra-ocular durante o procedimento não afetou a ferida pré-existente, tanto no caso do paciente submetido a transplante de córnea quanto nos submetidos à cirurgia de catarata.

Alterações endoteliais após LASIK têm sido estudadas ${ }^{(4-7)}$. Alguns autores ${ }^{(4)}$ acreditam que a perda endotelial de até 4,11\% relativa à quantidade de correção programada foi causada, provavelmente, pelas ondas de choque da fotoablação e pela manipulação corneana durante a ceratomileusis. Outros estudos, no entanto, tem demonstrado não haver alterações significativas no endotélio corneano após LASIK ${ }^{(1-3)}$. Nossos dados mostram que após 6 e 36 meses da cirurgia, a perda de 1,4\% e $2,67 \%$ observadas, respectivamente, na população das células endoteliais, não foram estatisticamente significativas, $(\mathrm{p}>0,05)$.

Quanto às complicações pós-operatórias, tínhamos a preocupação de que o risco de deslocamento traumático do disco corneano ou infecções fosse aumentado, simplesmente pelo fato de que crianças e adolescentes têm maior dificuldade em manter os cuidados pós-operatórios, o que não ocorreu em nenhum dos pacientes. Diferentemente de outros autores ${ }^{(14)}$, não observamos "haze" em nenhum caso. Dois pacientes referiram sintomas de olho seco durante a primeira semana após a cirurgia e foram tratados com lágrimas artificiais sem conservante. Os pacientes 7 e 9, que apresentaram, respectivamente, crescimento epitelial na interface e pregas na membrana de Bowman, não necessitaram de tratamento. O sucesso na redução da miopia e do astigmatismo miópico foi independente do erro refracional pré-operatório.

Três anos após a cirurgia, um pequeno aumento no erro refracional, com tendência à miopia, foi observado em 7 pacientes, o que provavelmente pode ser explicado pelas alterações refrativas naturais da idade e/ou regressão do efeito cirúrgico. Este aumento não atrapalhou nossos objetivos quanto ao uso dos óculos. A anisometropia máxima encontrada foi de 1,5 D e assim, o uso da correção visual adequada continuou viável para todos os pacientes que dela necessitassem. Cinco dos 9 pacientes permaneceram usando óculos enquanto que os outros 4, apresentavam AVSC de 20/50 ou melhor e não melhoravam com correção, devido à ambliopia.

A redução substancial encontrada na paquimetria foi semelhante àquela encontrada em estudos para correção de erros refracionais semelhantes em adultos ${ }^{(5)}$.

É difícil comparar este grupo heterogêneo de pacientes tão jovens, com outros grupos descritos na literatura. Estamos apenas iniciando nossos trabalhos, mas os resultados são encorajadores e reforçam os resultados descritos por outros autores $^{(12-14)}$. Com base nesses dados, poderíamos também pensar em realizar a técnica LASIK em pacientes com idade inferior a 18 anos, portadores de miopia bilateral moderada e córnea com espessura satisfatória, tendo como preocupação o fato de que, caso o erro refracional progrida até o início da idade adulta, um re-tratamento poderia ser realizado, assim como descrito para pacientes adultos ${ }^{(16)}$. 


\section{CONCLUSÕES}

Baseada em objetivos terapêuticos, a técnica LASIK, unilateral, realizada em crianças de 8 a 15 anos de idade, portadores de alta anisometropia, mostrou-se segura, eficaz e previsível, três anos após a cirurgia. O pequeno aumento encontrado na miopia ao longo do tempo, não inviabilizou o uso da correção visual adequada.

Estudos com maior número de casos e seguimento mais prolongado irão nos fornecer informações preciosas sobre a questão. Por enquanto, manteremos nossas indicações restritas a casos de alta anisometropia que não tenham obtido sucesso com os tratamentos convencionais.

\section{ABSTRACT}

Purpose: To determine the safety, efficacy, predictability and stability of laser in situ keratomileusis (LASIK), three years after surgery, to correct high myopia or high myopic astigmatism in children with high anisometropia in whom conventional treatments had failed. Methods: Nine eyes of 9 patients, 3 boys and 6 girls with a mean age of 11.5 years (range, 8 to 15 years) underwent LASIK using the Chiron Technolas 217 excimer laser. Minimum follow-up was 36 months. Results: Three years after LASIK, uncorrected visual acuity improved by at least five Snellen lines in all eyes; five eyes $(55.5 \%)$ were $20 / 50$ or better without correction. Six eyes $(66.6 \%)$, were $20 / 50$ or better with correction and five eyes $(55.5 \%)$ improved at least 1 line in the best spectacle corrected visual acuity (BSCVA). Due to amblyopia, no eye had uncorrected visual acuity of 20/20 or better. The mean spherical refraction equivalent decreased from -7.66 $( \pm 3.75)$ $\mathrm{D}$ to $-1.02( \pm 1.26) \mathrm{D}$, and mean preoperative astigmatism decreased from $-3.11( \pm 2.09)$ D to $-0.75( \pm 0.25)$ D. Highest anisometropia was 1.5 D. Conclusion: Three years after surgery, LASIK seems to be a safe and effective option for the correction of high anisometropia in children from 8 to 15 years old, in whom conventional therapy had failed. The natural change in refraction related to maturation did not affect the use of appropriate spectacle correction.
Keywords: Keratomileusis laser in situ; Myopia/surgery; Astigmatism/surgery; Treatment outcome; Adolescence; Child

\section{REFERÊNCIAS}

1. Pallikaris IG, Papatzanaki ME, Stathi EZ, Frenschock O, Georgiadis A. Laser in situ keratomileusis. Lasers Surg Med 1990;10:463-8

2. Ibrahim $\mathrm{O}$. Laser in situ keratomileusis for hyperopia and hyperopic astigmatism. J Refract Surg 1998;14(2 Suppl):S179-82.

3. Barbosa, RL, Nassaralla BRA. LASIK para anisometropia associada à ambliopia em pacientes adultos. Rev Bras Oftalmol 2001;60:97-103.

4. Pallikaris IG, Siganos DS. Excimer laser in situ keratomileusis and photorefractive keratectomy for correction of high myopia. J Refract Corneal Surg 1994;10:498-510.

5. Pallikaris IG, Siganos DS. Laser in situ keratomileusis to treat myopia: early experience. J Cataract Refract Surg 1997;23:39-49.

6. Nassaralla BR, Nassaralla JJ. Laser in situ keratomileusis after penetrating keratoplasty. J Refract Surg 2000;16:431-7.

7. Parisi A, Salchow DJ, Zirm ME, Stieldorf C. Laser in situ keratomileusis after automated lamellar keratoplasty and penetrating keratoplasty. J Cataract Refract Surg 1997;23:1114-8.

8. Donnenfeld ED, Kornstein HS, Amin A, Speaker MD, Seedor JA, Sforza PD, et al. Laser in situ keratomileusis for correction of myopia and astigmatism after penetrating keratoplasty. Ophthalmology 1999;106:1966-75.

9. Nano HD, Muzzin S, Irigaray F. Excimer laser photorefractive keratectomy in pediatric patients. J Cataract Refract Surg 1997;23:736-9.

10. Singh D. Photorefractive keratectomy in pediatric patients. J Cataract Refract Surg 1995;21:630-2.

11. Alió JL, Artola A, Claramonte P, Ayala MJ, Chipont E. Photorefractive keratectomy for pediatric myopic anisometropia. J Cataract Refract Surg 1998; 24:327-30.

12. Rashad KM. Laser in situ keratomileusis for myopic anisometropia in children. J Refract Surg 1999;15:429-35.

13. Rybintseva LV, Scheludchenko VM. Effectiveness of laser in situ keratomileusis with the Nidek EC-5000 excimer laser for pediatric correction of spherical anisometropia. J Refract Surg 2001; 17(2 Suppl):S 224-8.

14. Agarwal A, Agarwal A, Agarwal T, Siraj AA, Narang P, Narang S. Results of pediatric laser in situ keratomileusis. J Cataract Refract Surg 2000;26: 684-9.

15. Bicas HEA. Ambliopia: vícios de refração como causas de deficiência visual. Arq Bras Oftalmol 1992;55:206-8.

16. Barbosa RL, Nassaralla Junior JJ, Nassaralla B. Retratamento após LASIK. Rev Bras Oftalmol 2001;60:629-35.

17. Spiritus M. Comment corriger un anisométrope? Bull Soc Belge Ophtalmol 1997;264:57-61.

18. Nassaralla BR, Nassaralla JJ. Laser in situ keratomileusis in children 8 to 15 years old. J Refract Surg 2001;17:519-24.

19. Dada T, Vajpayee RB, Gupta V, Sharma N, Dada VK. Microkeratomeinduced reduction of astigmatism after penetrating keratoplasty. Am J Ophthalmol 2001;131:507-8.

20. Zadok D, Maskaleris G, Garcia V, Shah S, Montes M, Chayet A. Outcomes of retreatment after laser in situ keratomileusis. Ophthalmology 1999; 106:2391-4.

\section{Ao enviar um artigo para publicação, leia ATENTAMENTE as instruções para autores, constante no final de cada fascículo.}

